\title{
Les radioprotecteurs chimiques: bilan et perspectives
}

\author{
M. FATOME * \\ (Manuscrit reçu le 21 janvier 1987)
}

\begin{abstract}
RÉSUMÉ
Les meilleurs composés radioprotecteurs sont toujours des dérivés d'aminothiols, le plus actif ètant le phosphorothioate WR 2721. Malgré une ètude clinique réalisée aux Etats-Unis avec ce composé en raison de son action faible ou nulle sur les cellules tumorales, leur application humaine présente des difficultés, la plus importante venant du risque d'apparition d'effets secondaires. Une amélioration nette de leur activité et une diminution de leur toxicité a été récemment obtenue avec de nouvelles associations de composés et, en ce qui concerne la voie orale, par leur incorporation dans des vecteurs. Leur mécanisme d'action est complexe et multiple. En dehors de la capture des radicaux libres, I'hypoxie et l'activation de la glutathion peroxydase sont certainement des facteurs importants. Ils n'ont pas d'action sur l'incidence de la nausée et du vomissement radioinduits dont la prévention reste difficile. En revanche, certains, tout au moins, semblent avoir un effet antimutagène et antinéoplasique même s'ils sont délivrés après l'irradiation.
\end{abstract}

\section{ABSTRACT}

The best radioprotective compounds are still aminothiols derivatives, the most active being the phosphorothioate WR 2721. In spite of clinical trials carried out in the United States with this compound because of its low or negligible effect on tumoral cells, their human application presents some difficulties, the most important coming from the risk of appareance of side-effects. An enhancing of their activity and a lowering of their toxicity have been recently obtained with new associations of compounds and, concerning the oral way, by their incorporation into carriers. Their action mechanism is complex and multiple. Except free radical scavenging, hypoxia and glutathion peroxydase activation are certainly important factors. They have no action on the incidence of radioinduced nausea and vomiting, the prevention of which remains difficult. On the other hand, at least some of them seem to have an antimutagenic and antineoplastic effect, even if given after irradiation.

* Centre de recherches du service de santé des Armées, division de radiobiologie et radioprotection, 1 bis, rue du Lieutenant Raoul Batany, 92141 Clamart. 
La radioprotection chimique consiste à diminuer les effets de l'irradiation par l'administration préalable de composés chimiques. II s'agit donc d'une chimioprophylaxie. Elle peut intéresser des équipes d'intervention ou être utilisée chez des sujets soumis à une radiothérapie.

Les recherches ont débuté vers 1945. Les premiers travaux s'étaient révélés prometteurs avec la mise en évidence de l'activité radioprotectrice de plusieurs substances parmi lesquelles la cystéamine reste le radioprotecteur de référence. Par la suite, l'intérêt suscité par ces recherches avait faibli, les résultats s'étant révélés assez décevants. II $a$, en effet, fallu attendre une vingtaine d'années pour voir apparaître des substances beaucoup plus efficaces, les phosphorothioates, synthétisés aux USA au Walter Reed Institute. Le plus actif connu sous le sigle "WR 2721 " ou sous le nom plus récent d'Etiophos (ou de Gammaphos en Europe de l'Est) a fait l'objet de très nombreuses études. Par la suite, la mise en évidence d'une activité radioprotectrice beaucoup plus faible de ce composé sur les cellules tumorales que sur les cellules saines a donné une nouvelle impulsion aux travaux de recherches et a conduit les USA à entreprendre des études cliniques. Enfin, l'accident récent de Tchernobyl a entraîné une nouvelle prise de conscience de l'intérêt possible de ces substances.

La meilleure façon d'évaluer le pouvoir radioprotecteur d'un composé consiste à déterminer le facteur de réduction de dose ou FRD qui est le rapport entre la dose d'irradiation létale à $50 \%$ des sujets protégés et celle des sujets témoins non protégés. II est parfois désigné sous le terme de facteur de modification de dose généralement utilisé pour les radiosensibilisants. Un autre facteur, l'index de protection, est parfois mentionné. II dépend du rapport entre la plus faible dose efficace du composé et sa dose létale à $50 \%$, la plus faible dose efficace étant celle qui entraîne un taux de survie au moins égal à $30 \%$ chez les sujets irradiés à la dose létale $100 \%$ des témoins.

\section{QUEL BILAN PEUT-ON ACTUELLEMENT ÉTABLIR?}

A) II existe des substances efficaces qui peuvent être classées en trois catégories:

- produits de synthèse;

- composés naturels;

- substances agissant comme modificateur de réponse biologique.

\section{Produits de synthèse}

Douze à quinze mille molécules ont été synthétisées aux USA, environ quatre mille l'ont été en France par divers laboratoires universitaires par l'intermédiaire de la Division des recherches et études techniques. Le nombre de synthèses effectuées ailleurs, en particulier au Japon et en Europe de l'Est, est difficilement évaluable. 
Les meilleurs composés de synthèse sont toujours les aminothiols et leurs dérivés. La molécule de base est la cystéamine ou $\beta$ - mercapto-éthylamine ou MEA dont l'effet radioprotecteur a été mis en évidence par Bacq en 1951 [1]. Sa structure chimique $\mathrm{H}_{2} \mathrm{~N}-\mathrm{CH}_{2}-\mathrm{CH}_{2}-\mathrm{SH}$ se retrouve dans celle de la plupart des radioprotecteurs connus. Administrée sous forme de chlorhydrate par voie intra-péritonéale ou intra-veineuse à dose élevée, quelques minutes avant une irradiation $X$ ou gamma, elle assure chez le mammifère un FRD de l'ordre de 1,5. Sa charge positive et sa taille $(0,5 \mathrm{~nm})$ lui confèrent certaines propriétés particulières lui permettant de se lier aux parties d'ADN non recouvertes par les histones, de se lier de façon électrostatique aux têtes polaires des phospholipides et de pénétrer en phase fluide dans les bicouches lipidiques de la membrane. La substitution d'un groupement méthyle sur le carbone proche du groupement thiol (SH) entraîne une baisse de la toxicité de la molécule d'un facteur environ égal à 2 , et un FRD plus élevé qui atteint 1,85 . On ne peut pas actuellement donner d'explication à cet effet.

Les phosphorothioates caractérisés par la phosphorylation du thiol (liaison $\mathrm{S}-\mathrm{PO}_{3} \mathrm{H}_{2}$ ) constituent les meilleurs radioprotecteurs connus. Le plus actif est l'acide S 2 (3 - aminopropylamino) phosphorothioïque ou WR 2721 dont la structure est $\mathrm{H}_{2} \mathrm{~N}-\left(\mathrm{CH}_{2}\right)_{3}-\mathrm{NH}-\mathrm{CH}_{2}-\mathrm{S}-\mathrm{PO}_{3} \mathrm{H}_{2}$. Administré dans les mêmes condıtıons que précédemment, il a chez le mammifère un FRD de 2,7 [19]. Le groupement phosphate doit jouer à la fois le rôle de protecteur du thiol et celui de vecteur de la molécule. Au niveau cellulaire, une phosphatase acide hydrolyse la liaison $S-P$ et transforme le phosphorothioate en thiol qui est le principe actif. Le rôle de la partie gauche de la molécule reste imparfaitement connu, mais participe à l'effet radioprotecteur, l'effet diminuant si sa longueur est modifiée. L'effet supérieur de ces composés peut s'expliquer par une augmentation de la concentration en thiol au niveau cellulaire.

Les thiazolidines de structure

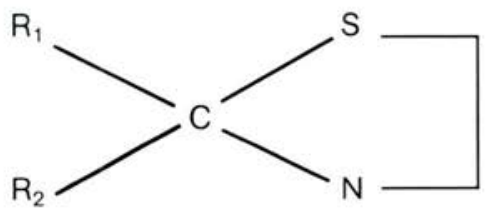

présentent un FRD égal à celui de la cystéamine, mais ont une durée d'action prolongée sur plusieurs heures et parfois une action retardée. Certaines présentent un effet notable à faible dose. Elles possèdent la structure de la cystéamine dans leur cycle et agissent in vivo par libération de ce composé à la suite d'une hydrolyse enzymatique. Les meilleures possèdent un groupement phényl en position $R_{1}$ [28]. Le remplacement du carbone substitué par $R_{1}$ et $R_{2}$ par du germanium ou du silicium a généralement entraîné une augmentation de l'effet radioprotecteur [25].

L'acétylation du groupement thiol de la cystéamine associée à la condensation sur son groupement aminé d'un acide aminé, comme la glycine, a conduit à des composés peu toxiques sans altération de l'effet radioprotecteur [17]. 
On peut encore citer d'autres familles chimiques comme les thiosulfates [4], les trisulfures à fonction sulfinate actifs à faible dose [4], les thiazolines [24], les benzonitriles [7], les adamantanes dont certains dérivés présentent une activité notable après leur administration orale, le meilleur ayant un FRD de 1,7 [32].

\section{Produits naturels}

La vitamine $E$ ou tocophérol, qui a un effet sur la peroxydation lipidique membranaire, possède un effet radioprotecteur lorsqu'elle est administrée pendant plusieurs jours.

La prostaglandine $\mathrm{PGE}_{2}$ sous forme diméthylée possède un effet protecteur avec un FRD qui peut atteindre 1,6. Des mélanges d'acides aminés, de vitamines et d'oligo-éléments présentent un léger effet.

\section{Substances agissant par modification de réponse biologique}

Certains polysaccharides extraits de levure présentent un FRD de 2. Ils agissent par stimulation du système hématopoïétique [16]. De même, des substances immuno-stimulantes comme le glucan présentent un effet notable.

Au terme de cette énumération non exhaustive, on peut constater qu'il existe des substances radioprotectrices.

B) Toutefois, la protection vis-à-vis du neutron est beaucoup plus difficile à obtenir, la plupart des radioprotecteurs connus n'ayant qu'un effet faible et le plus souvent négligeable. Même le WR 2721 n'a qu'une activité modeste avec un FRD de l'ordre de 1,25. En revanche, son dérivé méthylé sur le groupement aminé terminal lui confère un effet supérieur avec un FRD de l'ordre de 1,6 [27], une toxicité légèrement plus faible et un effet à peine inférieur vis-à-vis du rayonnement gamma (FRD 2,3). La raison de cet effet supérieur vis-à-vis du neutron reste inconnue.

C) De plus, l'application des radioprotecteurs à l'homme rencontre plusieurs difficultés qui sont liées aux constatations suivantes:

- L'effet radioprotecteur diminue généralement rapidement lorsque l'on diminue la dose administrée. II en résulte que l'obtention d'une radioprotection notable nécessite la délivrance d'une dose de composé susceptible d'entraîner des effets secondaires.

- II diminue généralement rapidement lorsque l'on augmente le délai compris entre l'administration et l'irradiation.

- Il est généralement faible ou nul lorsque l'administration est faite par voie orale.

Comme c'est le cas dans bien d'autres domaines thérapeutiques, ces inconvénients peuvent être en rapport avec une dégradation rapide du composé, une fois qu'il est introduit dans l'organisme. En particulier, les aminothiols subissent rapidement une dégradation oxydative qui peut être 
réversible sous l'effet d'une monooxygénase liée aux membranes ou irréversible sous l'effet d'une dioxygénase [35]. Introduits par voie orale, ils sont dégradés dans l'estomac tandis que les phosphorothioates sont transformés en thiols par hydrolyse acide.

Pour tenter de diminuer ces inconvénients, deux solutions sont actuellement envisagées:

- incorporer le radioprotecteur dans des vecteurs;

- pratiquer des associations.

\section{Incorporation des radioprotecteurs dans des vecteurs}

L'incorporation des radioprotecteurs dans des formes d'administration spécialement mises au point a pour objectif la protection et la libération prolongée de ces molécules entre leur lieu d'administration et leur site d'action. En raison du caractère très hydrophile et très peu lipophile de ces composés, notamment des phosphorothioates, la mise au point de ces vecteurs a souvent nécessité des aménagements des techniques classiques. Nous avons ainsi mis en évidence que, si la cystéamine est administrée par voie orale sous forme incorporée dans des liposomes, c'est-à-dire dans des vésicules de phospholipides et de cholestérol, elle présente alors un FRD de 1,4 sur 3-4 heures, alors qu'elle n'a pas d'activité si elle est délivrée par cette voie sous forme de solution dans l'eau [12, 22]. Plus récemment, nous avons constaté que, si le WR 2721 est administré par voie orale sous forme incorporée dans des microsphères d'éthylcellulose, i! présente un FRD de l'ordre de 1,8 sur une durée de $2-3$ heures [6]. Ces résultats montrent qu'il est possible d'améliorer de façon importante l'activité de radioprotecteurs par leur incorporation dans des formes galéniques. II a, de plus, été constaté, une baisse notable de la toxicité pouvant faire espérer que ces techniques peuvent également conduire à une diminution des effets secondaires. Ces études sont actuellement poursuivies.

\section{Associations}

Elles peuvent être pratiquées soit entre radioprotecteurs, soit avec d'autres substances.

Depuis de nombreuses années, il a été montré que l'association de radioprotecteurs peut entraîner un effet beaucoup plus important que lorsqu'ils sont administrés séparément.

Dans ce sens, l'association de composés actifs à faible dose ou celle de substances agissant différemment, comme le WR 2721 et la prostaglandine $\mathrm{PGE}_{2}$ ou comme le WR 2721 et le glucan peuvent se révéler intéressantes. L'association à d'autres composés peut également être bénéfique. II y a quelques années, nous avons montré que l'association du Diazépam qui est légèrement radioprotecteur et de la cystéamine conduit à une baisse de la toxicité de cette dernière et à une augmentation de son effet radioprotecteur [5]. Actuellement, des études américaines ont pour objectif la recherche de la diminution de toxicité du WR 2721 et de l'augmentation de son action à faible dose par son association à des composés, tels des sels 
de sélénium, susceptibles d'augmenter l'activité de la glutathion peroxydase, le glutathion étant considéré comme le principal radioprotecteur endogène. Les premiers résultats s'avèrent encourageants.

\section{D) Le WR 2721 est actuellement le seul radioprotecteur à faire l'objet d'études cliniques}

Ces études conduites depuis quelques années aux USA sont actuellement en phase II. Leur origine vient de la mise en évidence de la protection faible ou nulle exercée par ce composé sur les cellules tumorales [18]. Quatre explications sont actuellement proposées [33-34]. Du fait de la moindre vascularisation des tumeurs, la pénétration du composé y serait faible. Cette pénétration active dans les cellules saines serait seulement passive dans les tumeurs. La déphosphorylation serait moins active que dans les cellules saines. Enfin, la radioprotection est plus efficace dans les cellules oxygénées que dans les cellules hypoxiques. Un autre avantage du WR 2721 pour la radiothérapie vient de son absence d'influence sur l'effet de radiosensibilisants comme le Misonidazole sur les cellules tumorales. De plus, il diminue la toxicité d'agents utilisés en chimiothérapie comme la cyclophosphamide ou le cis-platine [13]. Au cours de ces études cliniques, il est administré par perfusion d'une durée de $15 \mathrm{~min}$ à la dose de 740 mg. $\mathrm{m}^{-2}$. Dans ces conditions, il entraîne des effets secondaires, notamment des vomissements dans environ $25 \%$ des cas et une hypotension légère et de courte durée dans environ $5 \%$ des cas. Les drogues associées auraient une influence sur l'incidence de ces effets. En revanche, il existe un effet bénéfique notable sur les tissus hématopoiétiques chez l'irradié global.

Il est à noter que le WR 2721 ne traverse pas la barrière hématoméningée et se trouve en concentrations négligeables dans le cerveau [29] dont la protection pourrait être assurée par des substances plus lipophiles comme les thiazolidines. Des études expérimentales sont actuellement conduites dans ce sens à Montpellier par le Prof. Chapat.

Enfin, d'autres substances que le WR 2721 ou les phosphorothioates sont capables d'exercer une protection plus faible sur les cellules tumorales. II en est ainsi de condensations d'acides aminés et de cystéamine S-acétylée [3].

\section{E) Prévention des effets tardifs}

Elle a été moins étudiée que celle des effets immédiats. Toutefois, plusieurs études réalisées par Maisin avec des radioprotecteurs seuls ou un mélange ont mis en évidence une diminution de l'incidence tumorale radioinduite [15].

Une étude américaine récente a montré une activité antimutagène et antinéoplasique du dérivé thiol du WR 2721 lorsqu'il est administré avant, pendant ou même après l'irradiation [9]. Cette dernière observation originale en soi n'a pas reçu d'explication pour l'instant. A priori, elle élargit le domaine d'intérêt de ces composés. 


\section{MĖCANISME D'ACTION DES RADIOPROTECTEURS}

Cet aspect fondamental des études de radioprotection chimique est essentiel à la découverte de nouveaux radioprotecteurs. Le mécanisme n'est pas encore parfaitement connu, même en ce qui concerne les aminothiols. II est en fait multiple [8].

Le mécanisme par capture de radicaux libres est considéré comme l'un des plus importants. II consiste en la capture de ces entités très réactives issues pour la plupart de la radiolyse des molécules d'eau de l'organisme et ayant pour effet la multiplication des lésions cellulaires induites directement par l'irradiation, en particulier au niveau des acides nucléiques et des membranes. Dans le même sens, la formation de ponts disulfure avec les protéines soufrées protège celles-ci de l'atteinte des radicaux libres.

Par ailleurs, le mécanisme par hypoxie est au moins aussi important [20]. L'oxygène est le principal radiosensibilisant, à l'origine des phénomènes de peroxydation. L'irradiation de cultures cellulaires sous saturation d'oxygène peut conduire à une augmentation de la radiosensibilité d'un facteur proche de 3 par rapport à l'irradiation sous azote. L'hypoxie diminue la peroxydation lipidique au niveau des membranes et semble favoriser les phénomènes de réparation des acides nucléiques [21]. II existerait un lien entre l'activité du WR 2721 et la réparation des acides nucléiques [23].

L'hypoxie peut être à l'origine des modifications du métabolisme cellulaire décrites par Bacq sous le terme général de "choc biochimique" à la suite de l'injection d'aminothiols. Ces modifications sont caractérisées par une diminution du mètabolisme oxydatif cellulaire, un arrêt provisoire des synthèses des acides nucléiques et une augmentation des réparations cellulaires. Le mécanisme de l'hypoxie par les aminothiols reste mal connu. Toutefois, des travaux récents que nous avons conduits avec le département de physicochimie du Centre d'études nucléaires de Saclay ont mis en évidence que la cystéamine entraîne une diminution importante de la diffusion de l'oxygène à travers les membranes artificielles, ceci sans consommation ni dégradation de la molécule [30].

II a, de plus, été montré que les radioprotecteurs soufrés, la cystéamine en particulier, se lient aux parties de la molécule d'ADN non recouvertes par les histones ainsi qu'aux têtes polaires des phospholipides [2, 31].

On ne saurait terminer ce chapitre sur le mécanisme d'action des radioprotecteurs sans évoquer l'effet sur le glutathion peroxydase. Le glutathion est, en effet, considéré comme le principal radioprotecteur endogène dont le rôle en tant que donneur d'hydrogène est particulièrement important. Plusieurs études récentes ont montré que la diminution de son taux intra-cellulaire par des substances comme la buthionine-sulfoximine entraîne une augmentation de la radiosensibilité cellulaire [26].

II semble exister une bonne corrélation entre le degré de radiorésistance et le niveau d'activité en glutathion peroxydase. La cystéamine, le WR 2721, le sélénium augmentent cette activité, alors que la vitamine $E$ en diminue la perte [10]. 


\section{REMARQUE}

Les radioprotecteurs n'ont pas d'effet sur le syndrome précoce des irradiés, et en particulier sur les nausées et les vomissements. La prévention de ces derniers reste difficile à obtenir, les antiémétiques connus ayant généralement un effet faible, inconstant ou nul et présentant souvent des effets secondaires indésirables. Leur étude implique une meilleure connaissance du mécanisme de déclenchement du vomissement radioinduit. Ce mécanisme est à la fois périphérique au niveau de l'estomac, et central au niveau de l'Area Postrema, structure qui possède plusieurs types de récepteurs spécifiques qui peuvent être stimulés par des substances véhiculées par le sang [11]. Les produits de dégradation libérés par l'irradiation restent encore mal connus. Les antiémétiques agissent généralement sur un type de récepteurs spécifiques et il est possible que leur association soit plus active, mais l'apparition d'effets secondaires risque alors d'être augmentée. De nouvelles substances sont actuellement étudiées en Grande-Bretagne et aux USA.

\section{CONCLUSION}

Les études de radioprotection chimique ont conduit à la mise en évidence de plusieurs composés efficaces. Toutefois, s'ils sont administrés isolément et sous forme de solution, le risque d'apparition d'effets secondaires indésirables aux doses susceptibles d'être efficaces, limite leur application à l'homme, notamment aux sujets faisant partie d'équipes d'intervention. En revanche, leur incorporation dans des vecteurs ou leur administration sous forme d'associations paraissent devoir faciliter cette application. Les études expérimentales actuellement réalisées dans ce sens, les études cliniques conduites chez l'homme aux USA, et la mise en évidence récente d'une action antimutagène et antinéoplasique exercée par le principe actif du meilleur radioprotecteur actuel, même s'il est délivré après l'irradiation, sont des éléments pouvant faire espérer la mise au point d'une radioprotection chimique efficace et bien tolérée.

\section{RÉFÉRENCES}

[1] BACQ Z.M., HERVÉ A., LECOMTE J., FISCHER P., BLAVIER J., DECHAMPS C., LEBIFIAN H., RAYET P. Protection contre le rayonnement $X$ par la $\beta$ - mercaptoétylamine. Arch. Intern. Physiol., 1952, 59, 442-447.

[2] BERLEUR F., ROMAN V., JASKIEROWICZ D., FATOME M., LETERRIER F., TERMINASSIAN-SARAGA L., MADELMONT G. The binding of the radioprotective agent cysteamine with the phospholipidic membrane head group - interface region. Biochem. Pharmacol., 1985, 34 (7), 3071-3080.

[3] BISCAY P., LESPINASSE F., OIRY J., HULZONSKI J., IMBACH J., MALAISE E.P. GUICHARD M. Radiobiological evaluation of a newly synthetized cysteamine derivatives. Int. J. Radiat. Oncol. Biol. Phys., 1986, 12, 1469-1473.

[4] DAVIDSON D.E., GRENAN M.M., SWEENEY T.R. Biological characteristics of some improved radioprotectors. In: Radiation sensitizers. Their use in the clinical management of cancer (L.W. BRADY, Ed.) New York: Masson, 1980, 309-318. 
[5] FATOME M., GAUDEFROY A., LAVAL J.D., ANDRIEU L., SENTENAC-ROUMANOU $\mathrm{H}$. Le Diazépam: action radioprotectrice et influence sur la toxicité et le pouvoir radioprotecteur de la cystéamine. Eur. J. Med. Chem., 1977, 12 (1), 93-95.

[6] FATOME M., COURTEILLE F., LAVAL J.D., ROMAN V. Radioprotective activity of ethylcellulose microspheres containing WR 2721, after oral administration. Int. J. Rad. Biol (sous presse).

[7] FERNANDEZ J.P., ROBBE Y., CHAPAT J.P., CASSANAS G., FATOME M., SENTENAC-ROUMANOU $\mathrm{H}$. Agents radioprotecteurs dérivés du benzonitrile. II Farmaco, 1986, 41 (1), 41-48.

[8] FOYE W.O. Mechanisms of radiation protection by the aminothiols. Int. J. Sulfur Chem., 1973,8 (1), 161-171.

[9] HILL C.K., NAGY B., PERAINO C., GRDINA D.J. 2 (Aminopropyl) amino ethanethiol (WR 1065) is anti-neoplastic anti-mutagenic when given during ${ }^{60} \mathrm{Co} \gamma-$ ray irradiation. Carcinogenesis, 1986, 7 (4), 665-668.

[10] HILL K.C., BURK R.F. Influence of vitamin E and selenium on glutathion - dependent protection against microsomal lipid peroxidation. Biochem. Pharmacol., 1984, 34, 1065-1068.

[11] ISON P.J., PEROUTKA S.J. Neurotransmitter receptor binding studies predict antiemetic efficacy and side-effects. Cancer Treat. Reports, 1986, 70 (5) 637-642.

[12] JASKIEROWICZ D., GENISSEL F., ROMAN V., BERLEUR F., FATOME M. Oral administration of liposome-entrapped cysteamine and the distribution pattern in blood, liver and spleen. Int. J. Radiat. Biol., 1985, 47 (6), 615-619.

[13] KLIGERMAN M.M. Phase I trial of WR 2721 in combination with radiation therapy, with the alkylating agents cyclophosphamide and cis-platinum. Cancer Clin. Trials, $1981,4,469-474$.

[14] KLIGERMAN M.M., GLOVER D.J., TURRISI A.T., NORFLEET A.L., YUHAS J.M., COIA L.R., SIMONE C., GLICK J.H., GOODMAN R.L. Toxicity of WR 2721 administered in single and multiple doses. Int. J. Radiat. Oncol. Biol. Phys, 1984, 10 (9), 1773-1776.

[15] MAISIN J.R., GERBER G.B., LAMBIET-COLIER M. Chemical protection against long-term effects of whole-body exposure of mice to ionizing radiation. $3-$ The effects of fractionated exposure to C 57 B1 mice. Radiat. Res., 1980, 82, 487-497.

[16] MAISIN J.R., KONDI-TAMBA A., MATTELIN G. Polysaccharides induce radioprotection of murine hemopoïetic stem cells and increase the LD 50/30 days. Radiat. Res., 1986, 105, 276-281.

[17] OIRY J., PUE J.Y., IMBACH J.L., FATOME M., SENTENAC-ROUMANOU H., LION C. Synthesis and radioprotective activity of new cysteamine and cystamine derivatives. J. Med. Chem., 1986, 29 (11), 2217-2225.

[18] PHILIPS T.L., KANE L., UTLEY J.F. Radioprotection of tumor and normal tissues by thiophosphate compounds. Cancer, 1973, 32, 528-535.

[19] PIPER J.R., STINGFELLOW C.R., ELLIOT R.D., JOHNSTON T.P. S-2- $(\omega$ aminoalkylamino) ethyldihydrogen phosphorothioates and related compounds as potential antiradiation agents. J. Med. Chem., 1969, 12 (2), 236-243.

[20] PURDIE J.W., INHABER E.R., SCHNEIDER H., LABELLE J.L. Interaction of cultured mammalian cells with WR 2721 and its thiol WR 1065: implications for mechanisms of radioprotection. Int. J. Radiat. Biol., 1983, 43 (5), 517-527. 
[21] QUINTILIANI M. The oxygen effect in radiation inactivation of DNA and enzymes. Int. J. Radiat. Biol., 1986, 50 (4), 573-594.

[22] ROMAN V., BOCQUIER F., LETERRIER F., FATOME M. Action radioprotectrice de la cystéamine incorporée dans des liposomes administrés par voie orale à la souris. C.R. Acad. Sc. Paris, 1982, 295, 191-193.

[23] RICKLIS E. DNA repair as a probe of radiosensitivity and radioprotection. In Radioprotectors and anticarcinogenes (O.F. NYGAARD and M.G. SIMIC, Eds.). New York: Academic Press, 1983, 363-380.

[24] ROBBE $Y$., FERNANDEZ J.P., CHAPAT J.P., SENTENAC-ROUMANOU $H$, FATOMEM. Recherche d'agents radioprotecteurs: 2-thiazolines et dérivés homologues. Eur. J. Med. Chem., 1985, 20 (1), 16-24.

[25] SATGE J., CAZES A., BOUCHAUT M., FATOME M., SENTENAC-ROUMANOU H. LION C. Synthèse et activité radioprotectrice de germa et silathiazolidines. Eur. J. Med. Chem., 1982, 17 (5), 433-436.

[26] VAN DER SCHANS G.P., VOS O., ROOS-VERHEIS W.S.D., LOHMAN P.H.M. The influence of oxygen on the induction of radiation damage in DNA in mammalian cells after sensitization by intracellular glutathion-depletion. Int. J. Radiat. Biol., 1986, 50 (3), 453-463.

[27] SIGDESTAD C.P., GRDINA D.J., CONNOR A.M., HANSON W.R. A comparison of radioprotection from three neutron sources and ${ }^{60}$ Co by WR 2721 and WR 151327. Radiat. Res., 1986, 106, 224-233.

[28] TEROL A., FERNANDEZ J.P., ROBBE Y., CHAPAT J.P., GRANGER R., FATOME M., ANDRIEU L., SENTENAC-ROUMANOU $\mathrm{H}$. Recherche d'agents radioprotecteurs dérivés de la phényl-2-thiazolidine. Eur. J. Med. Chem., 1978, 13 (2), 149-151.

[29] UTLEY J.F., MARLOWE C., WADDELL W.J. Distribution of S-35 labelled WR 2721 in normal and malignant tissues of the mouse. Radiat. Res., 1976, 68, 284-291.

[30] VACHON A., LECOMTE C., BRAQUET P., ROMAN V., FATOME M., BERLEUR F. Oxygen diffusion-concentration in phospholipidic model membranes. An ESRsaturation study. J. Chem. Soc. (sous presse).

[31] VASILESCU D., BROCH H., RIX-MONTEIL M.A. Mechanism of aminothiol radioprotectors action at the molecular level. J. Mol. Struct. (Theochem.), 1986, 134, 367-380.

[32] VOS O., ROOS-VERMET W.J.O. Protection against X - irradiation by some orally administered compounds. Int. J. Radiat. Biol., 1984, 45 (5), 475-486.

[33] YUHAS J.M., DAVID M.E., GLOVER D., BROWN D.Q., RITTER M. Circumvention of the tumor membrane barrier to WR 2721. Adsorption by reduction of drug hydrophilicity. Int. J. Radiat. Oncol. Biol. Phys., 1981, 8 (3-4), 519-522.

[34] YUHAS J.M., PHILLIPS T.L. Pharmacokinetics and mechanism of action of WR 2721 and other protective agents. In: Radioprotectors and anticarcinogens (O.F. NYGAARD and M.G. SIMIC, Eds.) New vork: Academic Press, 1983, 639-653.

[35] ZIEGLER D.M., POULSEN L.L., RICHERSON R.B. Oxidative metabolism of sulfurcontaining radioprotective agents. In: "Radioprotectors and anticarcinogens". (O.F. NYGAARD and M.G. SIMIC Ed.): New York: Academic Press, 1983, 191-202. 